\title{
The importance of staff's attitudes in the care of individuals with mental health problems in the emergency department
}

\author{
Marco Piccinelli \\ Department of Mental Health and Addictions, ASST Sette Laghi, Varese, Italy
}

\begin{abstract}
Individuals attending the emergency department due to mental health problems account for a significant proportion of all contacts, with an estimate of between $4 \%$ and $12 \%$. Numbers are expected to rise over time, straining the emergency care system in many countries. ${ }^{1-3}$

The specific needs of individuals with mental health problems are often challenging for the emergency department. Indeed, low confidence levels are often reported by the emergency department staff in the assessment of individuals with mental health problems at triage, a significant proportion of these individuals are seen in a timeframe which is rated as unsuitable for their condition and the lack of a quiet non-stimulating environment, together with frustration over long waiting time, might be demanding for them and produce emotional distress and behavioural discontrol.

The main issues related to presentations for mental health problems at the emergency department were investigated at a metropolitan hospital in Australia and the patient's journey of care in the emergency department was documented. Factors likely to be improved included availability of separate spaces to ensure privacy during mental health triage and assessment, better communication between hospital staff and patients regarding plans of care, and timely evaluation and treatment by a psychiatrist. $^{1}$ In another study, three key issues emerged when clinician perspectives were collected to improve a flow strategy at the emergency department for individuals with mental health problems: i) a care provision gap, due to lack of shared understanding between departments, insufficient education and limited process consistency; ii) misunderstandings about ownership of individuals with mental health
\end{abstract}

Correspondence: Marco Piccinelli, Department of Mental Health and Addictions, ASST Sette Laghi, Varese, Italy.

E-mail: marcopiero.piccinelli@asst-settelaghi.it

Key words: Mental disorders; Emergency department; Guidelines; Staff's attitudes; Violence.

Conflict of interest: the author declares no potential conflict of interest.

Funding: none.

Received for publication: 2 May 2019.

Accepted for publication: 3 May 2019

This work is licensed under a Creative Commons Attribution 4.0 License (by-nc 4.0).

CCopyright M. Piccinelli, 2019

Licensee PAGEPress, Italy

Emergency Care Journal 2019; 15:8261

doi:10.4081/ecj.2019.8261 problems in the emergency department; iii) dissonance in expectations of quality and timely care, associated with physical and mental health co-morbidities, organisational barriers and communication deficits. ${ }^{4}$

In light of these considerations, it is not surprising that great efforts have been placed on the development of guidelines helping to improve recognition of the current state of individuals with mental health problems at triage, to assign priority of interventions accurately and to define proper pathways to care at the emergency department. This is expected to be a never-ending process, deserving ongoing mutual collaboration between different health departments and staff members. As such, this situation is not to be viewed as frustrating or disappointing, since guidelines by their own are expected to require continuous support interventions to be implemented adequately and regular refinement to face the evolving needs of clinical practice.

There is no question about the fact that an effective partnership across different departments is expected to be critical in providing co-ordinated care for people who turn to the emergency department for a mental health problem and such collaboration across services and facilities plays a critical role on patient's perception of his/her recovery journey in the emergency department. When implementation of shared guidelines and mutual collaboration between health departments (specifically, the emergency and mental health departments) are discussed, professional knowledge, individual skills and organizational issues are easily identified as important variables to be improved. Instead, it is far more delicate and not straightforward to deal with staff's attitudes towards individuals with mental health problems presenting at the emergency department, although they deeply influence the application of guidelines.

In 2014, Clarke et al..$^{5}$ performed a literature review on emergency department staff attitudes towards mental health consumers. In general, caring for individuals with mental health problems turned out to be a costly challenge for the emergency department staff in emotional and practical terms. The general tenor of the literature was one of negative attitudes, overriding compassion and empathy that were also reported. In addition to fear and anger stimulated by aggressive or bizarre behaviour, significant staff's frustration and tension were sustained by the revolving door nature of many presentations, while the perceived lack of long-lasting results lead to a sense of hopelessness and a why bother? attitude. Moreover, individuals with mental health presentations tended to be perceived as manipulative and their complaints were often seen as not genuine on initial presentation.

It is interesting to note that, alongside with staff's personal variables (age, years of experience, skill level, perceived self-efficacy), the environmental climate of the emergency department might influence staff's negative attitudes towards consumers with mental health problems. Indeed, it has been repeatedly reported that the emergency department, as it is commonly configured, is perceived as inappropriate for people with mental health issues to attend in general, resulting in high levels of distress among staff members in dealing with these individuals. Frequent concerns 
referred to the high-pressured, high-stimulation, noisy and fastpaced environment of the emergency department, with lack of privacy and inadequate space and time preventing adequate care and producing frustration. Insufficient resources in the emergency department, including long waiting times, lack of specific mental health protocols/triage tools, the perceived lack of effective interventions, role ambiguity among health-care providers, and the continuous need for vigilance and control on the part of the staff in order to prevent violence or risk to self were perceived as draining and demotivating.

In general, the bad piece of news is that the greater is the negative affect of the emergency department staff towards individuals with mental health problems and the less is the propensity to help. The good piece of news is that educational interventions turn out to be effective in changing personal attitudes and, in some instances, staff with the most negative attitudes were those showing the most significant improvement.

The importance of staff's attitudes is confirmed by the observation that triage procedures might take greater advantage from awareness of cognitive and behavioural processes enacted by the emergency department staff than from strict compliance to guidelines and decision support tools. ${ }^{6}$ In this regard, a so-called mindful triage might be expected to improve the quality of care of individuals with mental health problems in the emergency department, by taking into account and assigning relevance to the cognitive aspects of the staff-patient encounter. By operating in this way, it might be reduced the tendency to simplify interpretations according to negative stereotypes held by health care professionals on individuals with mental health problems, improved sensitivity to clinical data and interventions in order to prevent diagnostic overshadowing (i.e., the default attribution of physical symptoms to the patient's mental disease and associated treatments, thus ignoring the potential occurrence of co-morbid organic pathology) and maintained a proactive attitude and alert attention to quickly detect and face unexpected events and outcomes. ${ }^{7}$

Talking about staff's attitudes, a final remark refers to the frequent occurrence of violent behaviours in the emergency department. Individuals with mental health problems may be involved, although many other patient and/or environmental factors are often operating. ${ }^{8}$ As a consequence of violence, staff members feel frightened and unease at the workplace and face burnout. In turn, this has a negative impact on staff's general productivity, ability to handle workload and cognitive demands, and provision of competent care and support. This is more likely to happen if violent events are perceived as an unavoidable part of the job, are not properly reported and do not receive adequate attention. This attitude has to be replaced by a safety culture through a clear definition and integration of clinical issues, environmental factors and legal elements that may prevent health care professionals from experiencing any form of violence at the workplace.

\section{References}

1. Morphet J, Innes K, Munro I, et al. Managing people with mental health presentations in emergency departments. A service exploration of the issues surrounding responsiveness from a mental health care consumer and carer perspective. Australasian Emerg Nurs J 2012;15:148-55.

2. Bost N, Crilly J, Wallen K. Characteristics and process outcomes of patients presenting to an Australian emergency department for mental health and non-mental health diagnoses. Int Emerg Nurs 2014;22:146-52.

3. Barrat H, Rojas-García A, Clarke K, et al. Epidemiology of mental health attendances at emergency departments: systematic review and meta-analysis. PLoS One 2016;11:e0154449.

4. Bost N, Johnston A, Broadbent M, Crilly J. Clinician perspectives of a mental health consumer flow strategy in an emergency department. Int Emerg Nurs 2015;23:265-73.

5. Clarke D, Usick R, Sanderson A, et al. Emergency department staff attitudes towards mental health consumers. A literature review and thematic content analysis. Int J Mental Health Nurs 2014;23:273-84.

6. Vogus TJ, Sutcliffe KM. The safety organizing scale: development and validation of a behavioural measure of safety culture in hospital nursing units. Med Care 2007;45:46-54.

7. Drach-Zahavy A, Saban M. Mindful triage: improving the quality of care of patients with mental illness in the emergency department. Int J Emerg Mental Health Human Resilience 2016;18:742-4.

8. Stowell KR, Hughes NP, Rozel JS. Violence in the emergency department. Psychiatr Clin North Am 2016;39:557-66. 